\title{
A CONSERVATION PLANNING APPROACH TO MITIGATING THE IMPACTS OF LEAKAGE FROM PROTECTED AREA NETWORKS
}

\section{AUTHORS:}

Michael Bode (corresponding author), Australian Research Council Centre of Excellence for Environmental Decisions, School of Botany, The University of Melbourne, Parkville, Melbourne, VIC 3010, Australia.

Email: mbode@unimelb.edu.au

Phone: +61 783460874

Ayesha Tulloch, Australian Research Council Centre of Excellence for Environmental Decisions, School of Geography, Planning and Environmental Management, The University of Queensland, Brisbane, QLD 4072, Australia.

Morena Mills, Global Change Institute, The University of Queensland, Brisbane, Queensland 4072, Australia University of Queensland, Brisbane, QLD 4072, Australia.

Oscar Venter, Centre for Tropical Environmental and Sustainability Science, and the School of Marine and Tropical Biology, James Cook University, Smithfield, QLD 4878, Australia.

Amy Ando, Department of Agricultural and Consumer Economics, University of Illinois at UrbanaChampaign, Urbana, IL 61801.

RUNNING HEAD: Conservation planning with leakage

ARTICLE LENGTH: 5998 words (incl. 235 word abstract); 5 figures; Supplementary methods.

KEYWORDS: Systematic conservation planning; reserve design; dynamic landscape modeling; household utility; habitat loss; socio-economic feedbacks; displaced effort. 
1 ABSTRACT

2 Protected area networks seek to restrict anthropogenic pressures in areas of high biodiversity. Resource users respond by seeking to replace some or all of the lost resources from locations elsewhere in the landscape. Protected area networks thereby perturb the pattern of human pressures by displacing extractive effort from within protected areas into the broader landscape, a process known as "leakage". The negative effects of leakage on conservation outcomes have been empirically documented and theoretically modeled using homogeneous descriptions of conservation landscapes. Human resource use and biodiversity vary greatly in space, however, and a theory of leakage must describe how this heterogeneity impacts the magnitude, pattern, and biodiversity impacts of leakage. In this paper we combine models of household utility, adaptive foraging, and biodiversity conservation to provide a bioeconomic model of leakage that accounts for spatial heterogeneity. We demonstrate 12 that leakage has strong and divergent impacts on the performance of protected area networks, undermining biodiversity benefits but mitigating the negative impacts on local resource users. Moreover, we find that when leakage is present, poorly designed protected area networks can result in a substantial net loss of biodiversity. We demonstrate how the effects of leakage can be mitigated if they are incorporated ex-ante into conservation decisions. Finally, if protected areas are coupled with non-reserve policy instruments such as market subsidies, we show that the trade-offs between 
Protected areas (PAs) are one of the planet's dominant land uses, covering more land area than all agricultural crops combined (UNEP-WCMC 2014). A primary benefit of PAs, and often the driving motivation for their establishment, is biodiversity conservation. However, PA networks are implemented within dynamic socio-ecological systems, where humans extract resources from the landscape to improve their welfare (Jakobsen 2006; Milner-Gulland 2011). If PAs successfully restrict access to resources, adaptive resource users may respond by seeking to replace them from elsewhere in the landscape (Ewers \& Rodrigues 2008). This process is known as "leakage", or "displaced effort", and is characterized by changes in human influences outside PA networks that are directly or indirectly attributable to those networks (Ewers \& Rodrigues 2008; Andam et al. 2008; Visconti et al. 2010).

Previous empirical work has shown that by increasing degradation rates outside of PAs, leakage negatively impacts unprotected biodiversity at local and regional scales (Andam et al. 2008; Ferraro \& Hanauer 2010; Meyfroidt et al. 2010). Additionally, economic models of how PAs change resource supply (Murray et al. 2004) and land availability (Armsworth et al. 2006) have also shown the harmful impacts of leakage. Particularly close attention, both theoretical and empirical, is paid to leakage in marine fisheries (generally called displaced effort), where the phenomenon is commonly included in bioeconomic analyses (Kellner et al. 2007). However, although bioeconomics has provided valuable insight into the dynamics of leakage, the primary focus has been on the amount of leaked habitat loss, not the co-location of that loss with heterogeneously distributed biodiversity features. Because of this, previous studies describe the impacts of leakage as a unidimensional, net displacement of effort from PAs to the unprotected landscape (e.g., Meyfroidt et al. 2010; Albers \& Robinson 2013; but see Visconti et al. 2010). However, patterns of both human resource use and biodiversity are heterogeneous and spatially patchy, as are the locations of PAs. The theory of systematic conservation planning, in particular, is primarily focused on choosing locations for PAs that best reflect the heterogeneous distribution of underlying biodiversity features. For a theory of leakage to incorporate and inform PA network design, it must therefore consider how resource use and 
biodiversity co-vary in a conservation landscape, and how their distributions influence leakage: its total amount, location, and impacts.

Here, we describe and construct the type of model required to incorporate leakage into the design of PA networks. This model integrates two bodies of theory to explain and predict the process and impact of leakage: natural resource economics, and systematic conservation planning. A number of studies have used broad conceptual and empirical models to investigate the simultaneous impacts of PA networks on biodiversity outcomes and poverty (Andam et al. 2010), and recent work has employed structural models of household behavior to predict spatial patterns of leakage (Albers \& Robinson 2013). However, no study has combined these, nor predicted how the behavior of local resource users might influence, and be influenced by, the distribution of biodiversity. Furthermore, although it is understood that leakage can undermine the benefits of PA networks and confound estimates of management effectiveness (Ewers \& Rodrigues 2008; Visconti et al. 2010), past work offers little guidance on how to design PA networks that will minimize or avoid the negative impacts of leakage. In this paper, we investigate how the design of PA networks shapes the amount and location of leakage, and through this process, the conservation of biodiversity and the welfare of local resource users.

We model a scenario where households from a local community are extracting a renewable resource from the surrounding local landscape for subsistence purposes. A conservation nongovernment organisation (NGO) is creating a network of no-take PAs to conserve local biodiversity; because this network will exclude resource users from some of the landscape, it will reduce the welfare of local resource users and create leakage. We use a landscape model to describe the location of PAs and the distribution of biodiversity, and to predict patterns of leakage and its biodiversity consequences. We couple this landscape model to a household utility model that predicts both the impact of the PA network on the welfare of local resource users, and how they will respond spatially to the new constraints on their resource supply. Finally, we model a set of three different, non-reserve policy instruments that the NGO could undertake to compensate local resource users for the impact of the PA network on household utility: a lump sum cash payment, an in-kind grant of the resource, and a 
market subsidy for the resource (or for a substitute). Through this coupled system model, we can predict the costs of these different policy instruments to the NGO, the effect they have on leakage, and the impact that each combination of PA network and instrument will have on biodiversity.

We use this system model to provide insight into three questions concerning the impacts of leakage on the performance of PA networks. First, how does the existence of leakage change our expectations about the net biodiversity conservation achieved by a given PA network, and its impacts on local resource users? Second, if leakage is a spatial response to constraints on resource extraction, (i) to what extent does leakage create conflict between biodiversity benefits and the welfare of resource users; (ii) how does the distribution of biodiversity in the landscape affect the severity of this conflict; and (iii) can a leakage-informed PA network avoid or reduce the need to trade these outcomes off against each other? Third, a range of non-reserve policy instruments can be used to compensate local communities for the opportunity costs imposed by PA networks. What is the relative impact of different non-reserve instruments on household utility and biodiversity conservation, and how do these different instruments affect the trade-off between the two objectives?

\section{METHODS}

To simulate the spatial dynamics of leakage, our system model integrates three elements: a spatial landscape model that contains heterogeneously distributed biodiversity, an adaptive foraging model of renewable resource extraction, and a model of household utility. While the resulting system model is structurally complex (Figure 1), each element is necessary to determine the extent and pattern of leakage, and the effect of management actions (both reserves and non-reserve policy instruments) on biodiversity conservation and the welfare of local resource users. In the sections that follow we describe the key dynamics of the three components of the system model, and then apply the results to a hypothetical linear landscape. A full mathematical definition of each of the component models, as well as the parameters chosen for the hypothetical system, is given in the supplementary methods.

\section{Model landscape}


The model dynamics take place in a linear landscape that contains a finite number of discrete parcels of land; each contains both a renewable resource and a quantity of biodiversity, both of which can be depleted by foraging effort (Figure 2). Foraging can only occur in parcels that have not been designated as protected areas by a conservation actor. Resource users are located in a community at one end of this landscape. Results from a linear landscape can be readily extended to two dimensions, resulting in radially symmetric patterns of foraging and habitat degradation.

One common example of a renewable resource is fuelwood, which accounts for $35 \%$ of energy supplies in the developing world and is a major source of forest degradation and the ensuing loss of biodiversity, ecosystem services, and erosion and flood protection (World Bank 1992). Like many renewable resources, fuelwood can be sourced from the landscape or purchased from a market. The household dynamics that determine the decision between harvesting or purchasing fuelwood are a focus of research in ecological economics (Heltberg et al. 2000).

\section{Household utility model}

The community contains a number of households, and each allocates a fixed amount of time between two different tasks: gathering the renewable resource from the landscape, and working for income (this latter option is equivalent to producing tradable goods). The households decide how to allocate their time in order to maximize their utility, which they can increase by consuming two types of goods: the renewable resource, and "other goods". The relationship between the amount of each good purchased and the benefit derived by the household is determined using a Cobb-Douglas utility function. This function yields household demand for each good that rises with income and falls with the price of the good. The function also implicitly assumes that households will tend to demand balanced bundles of goods rather than a bundle in which they spend all their income on one thing or another, but it does not implicitly constrain households to demand goods in fixed proportions. The Cobb-Douglas function does not assume that households have minimum threshold requirements for either of the goods, although variations of the function can include this (Supplementary methods). Households source the renewable resource by either foraging in parts of the landscape that have not 
been designated as protected areas, or by purchasing from the market. Other goods can only be obtained from the market.

\section{Optimal renewable resource foraging model}

The amount of the renewable resource that can be obtained from the landscape is determined using an optimal foraging model. Each parcel of land contains an equilibrium amount of the resource, which represents the balance between the rate of density-dependent (logistic) accumulation, and the foraging effort applied by the households. Each additional unit of foraging effort applied to a parcel requires a constant investment of time. However, the resource accumulation dynamics mean that increasing effort delivers diminishing marginal harvests. Foraging also involves travel time, proportional to the distance to the land parcel, and to the amount of effort applied there (based on the assumption that the foraging occurs over a large number of trips). Foraging effort will therefore be naturally concentrated on land parcels that are close to the community. Households allocate their foraging time budget across each of the non-reserved land parcels to maximize their total resource yield.

\section{Biodiversity conservation model}

We represent the amount of biodiversity in each parcel of land by a unidimensional quantity. Biodiversity is heterogeneously distributed through the landscape, and we select the amount in each parcel at random, from a uniform distribution. This biodiversity is negatively affected by foraging, proportional to the amount of effort applied to a particular parcel. Conservation actors are able to prohibit foraging in protected parcels, where the biodiversity will remain at its pristine level. The management objective is to maximize the sum of the biodiversity in protected and unprotected parcels, once the households have redistributed their effort in response to the new PA network.

\section{Modeling the effects of leakage}

The process, amount, and impacts of leakage are endogenous to the combined dynamics of the system model. The creation of a PA network forces resource users to re-optimize their harvesting decisions in three ways, simultaneously. First, they will adjust how much of the resource they use in 
total. Second, they will reconsider the proportions of the renewable resource that they source from the landscape and from the market, to maximize their household utility. Third, they will adjust their distribution of extractive effort through space, responding to the new constraints created by the PA network. The new effort distribution will affect the biodiversity outcomes achieved by the PA network, calculated by comparing the long-term average states of the harvested system before and after the protected areas are designated. That is, we are not considering the route by which the system approaches these equilibria, or the speed at which the system equilibrates.

\section{Analyses}

We apply three different analyses to this system model, to address each of our three primary questions. Our first analysis considers how the presence of leakage will change our expectations about the costs (to local households) and the benefits (to biodiversity conservation) of creating a PA network. To answer this question, we begin by calculating the equilibrium distribution of foraging effort that would exist in the absence of any PAs (the "no-reserve" case). Then, we implement a random PA network in the landscape, and calculate how households would re-distribute their effort around these new constraints (the "reserves-with-leakage" case). Finally, we define a third effort distribution, where we simply assume that when reserves are added to the no-reserve case, the effort distribution remains the same in unprotected parcels, but reduces to zero in protected parcels (the "reserves-without-leakage" case). Time that would have been spent foraging in protected parcels is spent working for income. To quantify how leakage will alter our expectations of the costs and benefits of PA networks, we calculate the changes in extant biodiversity and household utility that result from PAs in the presence of leakage (reserves-with-leakage, compared with no-reserves), and compare it to the naïve expectations in the absence of leakage (reserves-without-leakage, compared with no-reserves). We calculate this difference for a large number of PA networks, and illustrate the results with 10 examples.

Our second analysis investigates the outcomes that can be achieved by a range of different PA networks in the presence of leakage. Specifically, we are interested in the amount of conflict between biodiversity outcomes and household utility, and the degree to which an ability to predict outcomes in 
the presence of leakage allows this conflict to be avoided (i.e., allows both objectives to be achieved

177 simultaneously). We first use the system model to calculate the household utility and biodiversity outcomes for every possible PA network, identified through exhaustive search. We then choose the best subset of these PA networks by identifying Pareto efficient options (a PA network is Pareto inefficient if another network performs better according to one objective, and as good or better according to the other objective). The shape of the resulting Pareto frontier can be used to understand the trade-off between biodiversity and household utility that is made unavoidable by the patterns of biodiversity and resource use in the landscape, and by the process of leakage. Our third analysis considers how non-reserve policy instruments change the biodiversity and household utility consequences of PA creation, and the impact that such non-reserve instruments can have on the trade-off between the two objectives. We consider three types of non-reserve instrument that the NGO can undertake to provide additional benefits for biodiversity, and to mitigate the negative impacts of no-take areas on household utility. The first non-reserve instrument is a lump sum payment to the households, which will increase the household budget and better allow households to acquire the resource from the market, rather than by foraging. The second instrument is an in-kind grant of the resource (or a substitute) to each household, which releases money in the budget to purchase other goods. The third is a subsidy that reduces the market price of the resource, making it easier for households to acquire the resource rather than forage for it (e.g., the NGO would pay $10 \%$ of any fuelwood purchased from the market). We note that the in-kind grant is made up of renewable resource (or a substitute) that is sourced from outside the modelled landscape. If the resources that made up this grant were purchased from a similar community, then this instrument would simply shift leakage to more distant locations. We therefore assume that the in-kind grant is either derived from a sustainable source, or is an environmentally benign substitute. For the fuelwood example, in-kind grants could be charcoal briquettes created from agricultural byproducts. implementing NGO is equal, and is equivalent to an hour's wages per household, per week. In all cases household decisions will readjust to the new conditions; given that the non-reserve instruments affect 
the household dynamics in different ways, each will have a unique impact on household utility and extant biodiversity despite their equivalent cost to the NGO. We apply each non-reserve instrument to a set of four PA networks that were identified as Pareto efficient in our previous analysis, calculating the impacts on biodiversity and household utility for each.

\section{RESULTS}

The patterns of leakage produced by our system model (Figure 2) are visually similar to theoretical intuition (Ewers \& Rodrigues 2008) and empirical measurements (Winrock International 2002; Oliveira et al. 2007). The optimal foraging model encourages households to extract resources from locations that can be cheaply accessed, and the creation of a PA network therefore displaces effort onto parcels of land that are both unprotected and close to the community. However, the efficiency with which the resource can be extracted from a land parcel declines as foraging intensity increases; thus at some point households will begin to increase the effort in more distant parcels, rather than accept diminishing returns in the closest parcels. In general, because the constraints imposed by PAs make resource access more costly, they reduce the total foraging effort (i.e., leakage is not complete). However, the landscape's heterogeneous distribution of biodiversity means that a decline in total foraging effort does not necessarily improve biodiversity outcomes.

The system model predicts that displaced effort is greatest near the boundaries of the new PAs (Figure 2). The dynamics of the renewable resource in each parcel are spatially independent, and so the observed pattern of increase near to the PA does not reflect the movement of that resource across its boundary (i.e., the "spillover" of mobile resources; Abesamis \& Russ 2005; MacDonald et al. 2012). Instead, effort that can no longer be applied within a PA is displaced onto the closest parcels, because those adjacent areas have similar accessibility characteristics to the protected land. The characteristic pattern of high leakage at PA boundaries can therefore be driven by similarities in access costs, and does not require the presence of mobile resources. biodiversity benefits and human welfare impacts of a PA network (Figure 3). When leakage is included in our model, PA networks result in lower biodiversity than would be predicted if leakage is ignored. 
However, the negative social impacts of PAs are not as extreme as would have been predicted. Leakage therefore mitigates the negative effects of PAs on household utility, while reducing the expected biodiversity benefits.

As explained above, PA networks will put constraints on household foraging decisions, and the result can therefore never be an increase in household utility. In contrast, because biodiversity is distributed heterogeneously, the biodiversity impacts of a PA network can be either negative or positive (Figure 4). Compared to an unprotected landscape, most PA networks improve landscape biodiversity, and all have a negative impact on household utility. However, a sizeable minority of the PA networks (12\%) lead to a net reduction in biodiversity (to the left of the vertical grey line) through the indirect effects of leakage. Larger PA networks are more likely to have large positive impacts on biodiversity, but also large negative impacts on household utility.

The specific examples shown in Figure 4 illustrate the range of outcomes on the Pareto frontier. Network 1 minimizes the impacts on households by protecting a small number of land parcels that are too far from the community to be exploited, but consequently achieves little net biodiversity benefit. Network 3 maximizes biodiversity benefits by protecting a large number of parcels, particularly those that have high biodiversity, and/or are heavily used by the community. However, this network imposes large costs on households. Network 2 represents a compromise by ensuring the protection of high biodiversity parcels, but avoiding the most accessible parcels that would drive high leakage. The final two example networks show how the creation of protected areas can result in net negative conservation outcomes. Both networks 4 and 5 target the most threatened parcels for protection, but fail to completely protect the adjacent, biodiverse parcels, upon which effort is displaced.

Figure 4 shows a conflict between the benefit of PAs for biodiversity and their impacts on human welfare; the two cannot be simultaneously optimized. Without an unambiguously optimal choice, decision-makers must trade biodiversity benefits against the costs to local households. However, while no single PA network satisfies both objectives, a large number are clearly suboptimal. For each PA network that is interior to the Pareto frontier (i.e., below and to the left of the red line), 
there is an alternative network on the frontier that would improve outcomes for biodiversity or household utility (or both), without a reduction in either. The shape of this Pareto frontier shows that trade-offs are most severe when networks are biased towards one or the other objective. For example, when PA networks are providing large benefits for biodiversity at a great cost to households (lower right on the frontier), considerable household utility gains can be obtained at small costs to biodiversity outcomes.

In our integrated system model, leakage is an endogenous process that emerges from the reequilibration of a coupled socio-ecological system. A mechanistic description of this process allows the model to predict how policy instruments that do not involve PA creation will nevertheless affect biodiversity outcomes, by explaining how they affect household utility. Figure 5 shows the dual impact (biodiversity and household utility) of three non-reserve instruments, applied to four Pareto-efficient PA networks. The non-reserve instruments consistently improve the utility of households who extract resources from the landscape. This outcome is expected, since all three mechanisms directly improve components of utility. However, the direction of the biodiversity impacts vary by mechanism and PA network. Where PA networks have small impacts on utility and small benefits for biodiversity (e.g., A or B; Figure 5), a coupled market subsidy has a large and positive impact on biodiversity; an in-kind grant has no effect on biodiversity; and a lump sum subsidy causes biodiversity declines. Where PA networks have large impacts on household utility and strong benefits for biodiversity (e.g., C or D), all three non-reserve instruments have a small impact on biodiversity, with the market subsidy being slightly better than in-kind grants or lump sum subsidies.

Lump sum subsidies (cash paid to resource users as compensation for lost access) have a negative impact on biodiversity because they increase total household consumption, but for our parameterisation this effect was negligible. In contrast, by directly providing households with extra resource, in-kind grants encourage households to spend more time pursuing other goods to rebalance their utility function. This will reduce extractive effort, and therefore improve biodiversity 
encourage households to source their resources from the market rather than the landscape, which directly reduces foraging.

\section{DISCUSSION}

Our results show that leakage alters both the positive and negative effects of PA networks in important and complex ways. By redistributing extractive effort across the landscape, leakage reduces the biodiversity benefits expected from PA networks. For this reason, accounting for leakage is critical for accurate and realistic predictions of conservation outcomes (Ewers \& Rodrigues 2008). Moreover, we find that acknowledging leakage can alter the rankings of different PA networks, and even change the biodiversity impact of some networks from positive to negative. Leakage must therefore be incorporated into systematic conservation planning methods, since it will affect the core purpose of these tools: to correctly rank network performance. Our results show that leakage impacts are contextual, which helps to explain contrasting empirical findings (e.g., (Andam et al. 2010) c.f., (Oliveira et al. 2007; Meyfroidt \& Lambin 2010)). However, despite the range of potential outcomes, a mechanistic understanding of leakage can allow conservation planners to avoid the worst biodiversity consequences of leakage.

Leakage has important effects on the utility of local communities who extract resources from the landscape. PA networks restrict resource access, but leakage allows communities to compensate by increasing their extractive effort in areas that remain unprotected. Attempts to minimise leakage from PAs will therefore increase their negative impacts on local communities. However, if this process of effort redistribution can be accurately predicted, our results show that Pareto efficient compromises between benefits to biodiversity and costs to household utility are available. These compromises will become difficult to achieve in landscapes where biodiversity and resource use is positively correlated (Visconti et al. 2010), for example, where the biodiversity provides or supports the resource (e.g., bushmeat). However, regardless of the correlation, the methods we describe would still be able to identify Pareto efficient options. 
create large amounts of leakage, incur large costs on households, or both; networks that protect the highest biodiversity parcels (i.e., maximize gain) can avoid leakage but will not avert loss. Such traditional approaches fail because they focus only on the characteristics of the parcels being protected. Leakage requires decision-makers to consider biodiversity not only in the parcels that will be protected, but also in the parcels that will remain unprotected. PAs should be directed towards locations where high biodiversity parcels are surrounded by lower value parcels, which are most likely to receive displaced effort. Leakage therefore demands a subtle but important reframing of systematic conservation planning, where the purpose moves beyond identifying a subset of important locations for protection, to identifying locations where access restrictions will redirect degradation onto relatively low biodiversity parcels, or towards market alternatives. Conservation planning should not attempt to only halt degrading activities, it should be attempt to direct them.

In our model, protected areas place local constraints on degrading processes, but do not alter their dynamics. For example, harvesting was not permitted in our protected areas, but this did not make it more costly for people to travel through the landscape. In contrast, protected areas often explicitly prohibit access, or make access more expensive by limiting infrastructure. This can increase the cost of foraging in the land parcels beyond protected areas, thereby creating barriers to the spread of degradation into the landscape beyond the protected areas (Peres \& Terborgh 1995; Barber et al. 2014). Conversely, protected areas can increase the value of nearby land, attracting degrading forces to the reserve boundaries (Radeloff et al. 2010). Such processes could be included into our system model using case-specific foraging models, and spatially-explicit landscapes which include factors such as heterogeneous travel-times and access networks.

Leakage results from the adaptive decisions of resource users, represented in our model by households. The importance of understanding the mechanistic relationship between biodiversity loss and resource use has been highlighted in the resource management (Milner-Gulland 2011) and conservation planning literatures (Klein et al. 2008), but this is the first study that considers - from a planning perspective - the local socio-economic feedbacks that result from different PA networks. As we have shown, a conservation planning approach that explicitly describes the decision-process of 
resource users has two key benefits. First, because resource extraction is a key threat to biodiversity, an understanding of this process is needed for accurate estimates of biodiversity benefit. Second, a model of household utility allows planners to predict not just the impact of PAs on resource users, but also their response to these constraints, both of which affect the net impact of conservation actions (Milner-Gulland 2011). Such a model also allows planners to estimate the amount of restitution that would be required to compensate local communities for the access restrictions created by PA establishment.

In addition to measuring the impact of PA networks on local communities, a mechanistic model of household utility allows conservation planners to evaluate the performance of non-reserve policy instruments, as we have demonstrated for three alternatives. PA networks reduce community access to resources, but individuals are better able to cope with these added constraints when alternative occupations or substitute resources are available or are provided (Cinner et al. 2009). The need to consider non-reserve instruments (e.g., forms of resource management, education, alternative livelihoods) has therefore been emphasized by the conservation literature (Venter et al. 2008) and organizations (Tucker 1999). To accurate predict the biodiversity benefits of such instruments, we will need a better understanding of how different communities and individuals will respond to alternatives or subsidies. However, as we have shown here, accurate prediction will also require an understanding of the leakage interactions between household dynamics and the conservation landscape.

Our system model comprises three coupled sub-models: a landscape model containing biodiversity and a renewable resource; an adaptive model of resource extraction and biodiversity degradation, and a model of household utility (Figure 1). Because our purpose was to investigate the interaction between leakage dynamics and spatially structured biodiversity, we chose straightforward and general sub-models for each of these processes, and did not attempt to parameterize a specific case-study. The result was a system model with complex interconnections, but simple individual components. For this model to move beyond exploration into quantitative prediction and prescription, each component would need to be elaborated, and adapted to the specific socio-ecological context. 
Relaxing a number of key assumptions could offer different dynamics and valuable insights into leakage. First, the household utility model ignored potential heterogeneity in households' dependency on a resource, or their response to non-reserve interventions, which can be caused by differences in income, education and access to markets (Mitra \& Mishra 2011). Second, we also assumed that market prices were fixed, and are unaffected by local changes in demand for market goods; future work could take a more general-equilibrium approach and allow prices to be endogenous, or allow for situations in which people gather products from the forest to sell in the market rather than to use for personal purposes. Third, removing the last, small amount of a logistically-renewable resource will be uneconomic, and harvesting is therefore sustainable in our model. Different renewable resource models that contained Allee effects or alternative stable states would exacerbate the impacts of leakage, since the concentration of displaced effort could collapse ecosystems or drive populations to extinction. Finally, we used a homogeneous, linear landscape that contained a single, spatiallyindependent resource. Future research should explore the robustness of the observed dynamics to renewable resources that are mobile (Abesamis \& Russ 2005; Macdonald et al. 2012); such analyses will profit from comparisons to the parallel fisheries literature.

\section{REFERENCES CITED}

Abesamis, R. A., and G. R. Russ. 2005. DENSITY-DEPENDENT SPILLOVER FROM A MARINE RESERVE: LONG-TERM EVIDENCE. Ecol Appl 15:1798-1812.

Albers, H. J., and E. J. Z. Robinson. 2013. A review of the spatial economics of non-timber forest product extraction: Implications for policy. Ecological Economics 92:87-95.

Andam, K. S., P. J. Ferraro, A. Pfaff, G. A. Sanchez-Azofeifa, and J. A. Robalino. 2008. Measuring the effectiveness of protected area networks in reducing deforestation. Proceedings of the National Academy of Sciences 105:16089-16094. National Acad Sciences.

Andam, K. S., P. J. Ferraro, K. R. E. Sims, A. Healy, and M. B. Holland. 2010. Protected areas reduced poverty in Costa Rica and Thailand. Proceedings of the National Academy of Sciences 107:999610001.

Armsworth, P. R., G. C. Daily, P. Kareiva, and J. N. Sanchirico. 2006. Land market feedbacks can 
undermine biodiversity conservation. Proceedings of the National Academy of Sciences 103:54035408.

Barber, C. P., M. A. Cochrane, and C. M. Souza Jr. 2014. Roads, deforestation, and the mitigating effect of protected areas in the Amazon. Biological ....

Cinner, J., M. Fuentes, and H. Randriamahazo. 2009. Exploring social resilience in Madagascar's marine protected areas. Ecology and Society 14:41.

Ewers, R. M., and A. S. L. Rodrigues. 2008. Estimates of reserve effectiveness are confounded by leakage. Trends in Ecology \& Evolution 23:113-116.

Ferraro, P. J., and M. M. Hanauer. 2010. Protecting Ecosystems and Alleviating Poverty with Parks and Reserves: “Win-Win” or Tradeoffs? Environmental and Resource Economics 48:269-286.

Heltberg, R., T. C. Arndt, and N. U. Sekhar. 2000. Fuelwood Consumption and Forest Degradation: A Household Model for Domestic Energy Substitution in Rural India. Land Economics 76:213.

Jakobsen, J. 2006. The role of NTFPs in a shifting cultivation system in transition: A village case study from the uplands of North Central Vietnam. Geografisk Tidsskrift-Danish Journal of Geography 106:103-114.

Kellner, J. B., I. Tetreault, S. D. Gaines, and R. M. Nisbet. 2007. Fishing the line near marine reserves in single and multispecies fisheries. Ecol Appl 17:1039-1054.

Klein, C. J., A. Chan, L. Kircher, A. J. Cundiff, N. Gardner, Y. Hrovat, A. Scholz, B. E. Kendall, and S. Airamé. 2008. Striking a Balance between Biodiversity Conservation and Socioeconomic Viability in the Design of Marine Protected Areas. Conservation Biology 22:691-700. Blackwell Publishing Inc.

Macdonald, D. W., P. J. Johnson, L. Albrechtsen, S. Seymour, J. Dupain, A. Hall, and J. E. Fa. 2012. Bushmeat trade in the Cross-Sanaga rivers region: Evidence for the importance of protected areas. Biological Conservation 147:107-114.

Meyfroidt, P., and E. F. Lambin. 2010. Forest Transition in Vietnam and Bhutan: Causes and Environmental Impacts 10:315-339. Springer Netherlands, Dordrecht.

Meyfroidt, P., T. K. Rudel, and E. F. Lambin. 2010. Forest transitions, trade, and the global 
Milner-Gulland, E. J. 2011. Integrating fisheries approaches and household utility models for improved resource management. Proceedings of the National Academy of Sciences 108:1741-1746.

Mitra, A., and D. K. Mishra. 2011. Environmental resource consumption pattern in rural Arunachal Pradesh. Forest Policy and Economics 13:166-170.

Murray, B. C., B. A. McCarl, and H.-C. Lee. 2004. Estimating Leakage from Forest Carbon Sequestration Programs. Land Economics 80:109-124. University of Wisconsin Press.

Oliveira, P. J. C., G. P. Asner, D. E. Knapp, A. Almeyda, R. Galván-Gildemeister, S. Keene, R. F. Raybin, and R. C. Smith. 2007. Land-use allocation protects the Peruvian Amazon. Science 317:1233-1236.

Peres, C. A., and J. W. Terborgh. 1995. Amazonian nature reserves: an analysis of the defensibility status of existing conservation units and design criteria for the future. Conservation Biology. 
444 Figure 1: Schematic representation of the leakage model, applied to a linear landscape of 10 patches 445 (shown on left with the access point marked: A). Model components are color-coded: the optimal foraging model (purple); the biodiversity conservation model (green); and the household utility model (red). Dashed lines indicate reserve and non-reserve actions that managers control. Double-line boxes represent decisions made by households (how much and where to forage) who attempt to maximise household utility. Arrows indicate model components that exchange information. The shaded grey region highlights the dynamics that drive leakage.

Figure 2: Modelled impacts of an protected area (PA) network in the presence of leakage. Landscape parcels are shown as bars along the $x$-axis, with the community located at $x=0$. The $y$-axis indicates the change in human impact before and after the PA networks have been created. The PAs (at $x=1$ and $x=2$, in the region marked "A") reduce local human impacts as intended. The first PA reduces human impacts by the largest amount because that parcel was the most intensely harvested. However, leakage causes an increase in human impacts beyond PA boundaries (in the unprotected region marked " $\mathrm{B}$ "). Human impacts in the landscape parcels for $x \geq 8$ are unchanged as these parcels are too distant for foraging to ever be economical.

Figure 3: Changes in household utility and extant biodiversity resulting from 10 randomly chosen protected area (PA) networks. Vertical dashed lines show outcomes without any protection. Darker colored bars show the predicted outcomes of PA networks when leakage is ignored; lighter bars show the realized outcome with leakage. Leakage reduces the benefits achieved for biodiversity, but reduces the costs imposed on households. Leakage alters the performance rankings of PA networks, measured according to either objective. It can also change a PA network from having a net positive impact on biodiversity to having a net negative impact.

Figure 4: Extant biodiversity (x-axis) and household utility ( $y$-axis) achieved by all possible protected area (PA) networks. Performance is reported relative to the outcomes without any PAs (black marker; grey dashed lines). Markers are color-coded by size of the PA network (red = 0-2 parcels; green = 3-5 
parcels; blue $=6+$ parcels). Light red line indicates the approximate position of the Pareto frontier. Five

470 markers are numbered in the left hand panel; the corresponding PA networks are shown in the right

471 hand panels. The bar height indicates the relative amount of biodiversity found in each parcel; and

472 green shading indicates protected parcels.

473 Figure 5: Effect of non-reserve policy instruments on extant biodiversity and household utility.

474 Performance is reported relative to the outcomes without any protected areas (PAs). Letters 475 correspond to four different PA networks, shown on the right. Letter colors indicate the outcomes of 476 the reserve-only action (black), and of PA networks plus three different non-reserve instruments 477 (green = lump sum payment; blue = in kind grant; red = market subsidy). The size of each non-reserve 478 instrument has been chosen to equalize the total costs to the NGO. 